\title{
The Linkage of Zlib to Teapot for Auto-Differentiation Map Extraction and Nonlinear Analysis*
}

\author{
N. Sun, Y.T. Yan, F. Pilat, and G. Bourianoff \\ Superconducting Super Collider Laboratory ${ }^{\dagger}$ \\ 2550 Beckleymeade Ave. \\ Dallas, TX 75237
}

May 1993

\footnotetext{
*Presented at the 1993 IEEE Particle Accelerator Conference on May 17-20, Washington, D.C.

†Operated by the Universities Research Association, Inc., for the U.S. Department of Energy under Contract No. DE-AC35-89ER40486.
} 


\title{
The Linkage of Zlib to Teapot for Auto-Differentiation Map Extraction and Nonlinear Analysis
}

\author{
Ning Sun, Yiton T. Yan, Fulvia Pilat, and George Bourianoff \\ Superconducting Super Collider Laboratory, 2550 Beckleymeade Avenue Dallas, TX 75237, USA
}

\begin{abstract}
The differential Lie algebraic numerical library, Zlib has been linked to Teapot, the accelerator simulator code. This makes possible the use of the operational correction features of Teapot to produce a corrected lattice, and then choose either map or thin element-by-element tracking for tracking studies. Thin-element tracking is more accurate but slower than map tracking; therefore, the option of choosing one or the other is very desirable.
\end{abstract}

\section{INTRODUCTION}

Element-by-element tracking with Teapot for the Superconducting Super Collider (SSC) (more than 20,000 thin elements) for a thousand turns takes many hours of Sun workstation CPU time. The results are used to analyze the properties of the lattice, such as linear aperture, resonances, and short-term stability. One way to reduce the computing time is to use vectorized or parallel computing such as Ztrack [1] and Hypertrack [2]; another way is to use a nonlinear mapping method to map the lattice, such as Automatic Differentiation maps, which have been applied to the study of the long-term stability of the SSC lattices with the use of Zlib [5] and its related programs [3], [4].

However, no attempt has been made so far to use maps for short-term tracking because the extraction of very high order maps takes a long time and therefore gives no advantage over direct tracking. However, experience shows that maps of relatively low orders give quite reliable results for the analysis of some features of the accelerator lattice. To take advantage of the fast speed of one-turn map tracking, we have started the implementation of Zlib directly in Teapot [6]. We present the preliminary results on the reliability and tracking speed of the low-order maps and show how to combine the tools of Zlib and Teapot for convenient comprehensive studies.

\section{MAP EXTRACTION AND TRACKING}

First, we take the collider lattice of 20,000 elements, extract maps of various orders, record the time it takes to extract each of them, track the particles, and compare the results with those obtained from the Teapot. The lattice we are using is the one with collision optics, including IR regions, all errors, corrections and crossing angle of $135 \mu \mathrm{rad}$ (3B specifications for alignment and multipole errors; triplets have $0.1 \mathrm{~mm}$ misalignment and $0.2 \mathrm{mrad}$ rotation errors). The time taken by the map extraction is proportional to the number of elements in the lattice and the number of monomials in the map, of which the latter is given by

$$
n m=(n v+n o) ! /(n v ! n o !)
$$

for a no-order and nv-variable map.

\footnotetext{
*Operated by the Universities Research Association, Inc., for the U.S.
} Department of Energy under Contract No. DE-AC35-89ER40486.
In Figure 1, the extraction and tracking times are plotted vs. map orders from 2 to 6 , with the Teapot tracking as a reference. We use a SUN Sparc2 workstation and take a typical case (for smear and linear aperture studies) in which $6 \mathrm{C}$ particles are tracked 1024 turns along the lattice. All maps are $6 \times 6$, i.e., 6-dimensional, 6-variable. We notice the following:

(1) The extraction and tracking time for Zmap of order 4 or 5 are significantly shorter than direct elementby-element tracking.

(2) Once a map has been obtained, tracking of 1,024 turns takes just a few minutes because the whole lattice is represented by one element (the map). This opens the possibility of tracking bunches of particles to get the detailed features of the phase space.

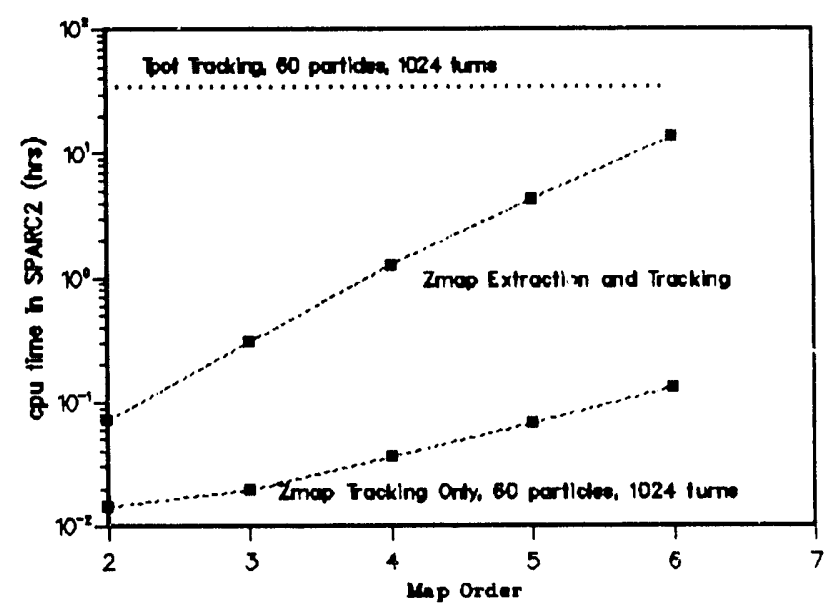

Figure 1. Speed of Zmap and Teapot tracking for the collider lattice.

\section{LINEAR AND DYNAMIC APERTURES}

All trackings are performed with respect to the closed orbits. The turn-by-turn data from every tracking case is analyzed to find the smear and tune shifts with amplitude so that the linear apertures (defined as occurrence of $5 \%$ smear or $0.5 \%$ tune shift, whichever comes first) can be determined. The results are shown in Figure 2 for the Collider Lattice; and results from Teapot direct tracking are plotted as references.

What we can see from these figures is that the 5 th order map is very accurate compared with the direct tracking; and the horizontal result is better than the vertical one for lower orders.

In Figure 3 we show linear apertures calculated from maps extracted for the SSC Medium Energy Booster lattice (MEB20-version). In this case, the convergence toward the results from direct tracking (shown as parallel horizontal lines in the figure) looks even more consistent with the increment of map orders. 
In Figure 4, the dynamic apertures calculated for the Collider and MEB lattices by Zmap and Teapot are plotted. Although one cannot expect by either method to obtain an accurate dynamic aperture from the tracking of only 1,024 turns, the map results are still in very good agreement with those from Teapot with the use of 5th order maps in both cases; and we expect to get even better agreement from map tracking if more than 1,024 turns are tracked: because as the dynamic aperture shrinks with longer turns, the map will definitely become more accurate.

$.10^{-3} \mathrm{~m}$

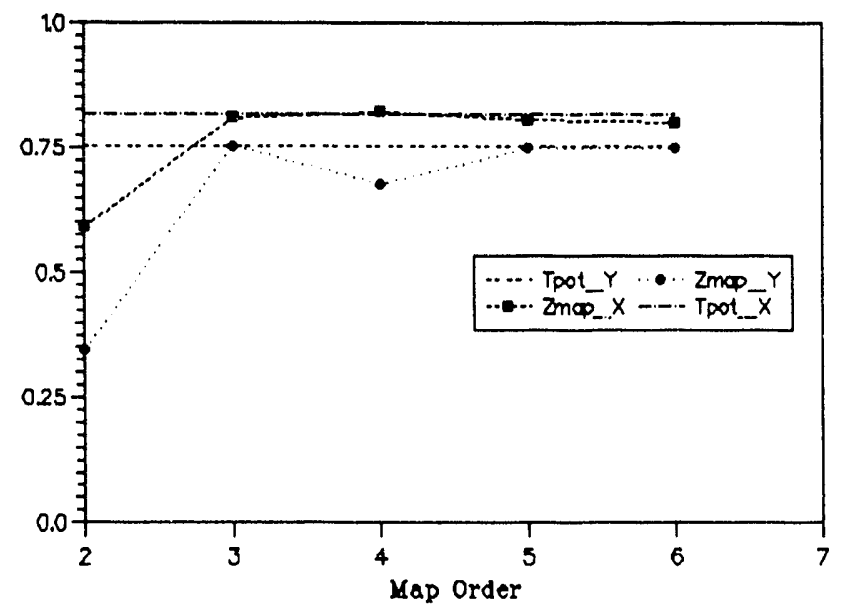

Figure 2. Horizontal (x) and vertical (y) linear apertures of the collider lattice.

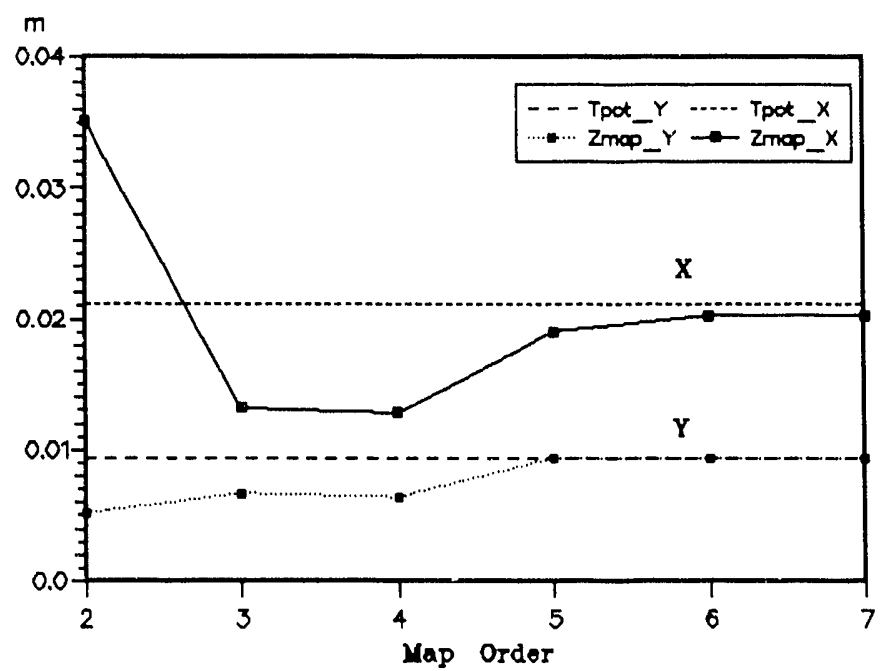

Figure 3. Horizontal ( $x$ ) and vertical (y) linear apertures of the MEE lattice.

\section{IMPLEMENTATION OF ZLIB IN TEAPOT}

Teapot and Zlib (and other Z-family programs) were originally independently written and compiled, with a nonlinear machine "Zfile" as the only readable input data structure of the Z-family program. Most variables were

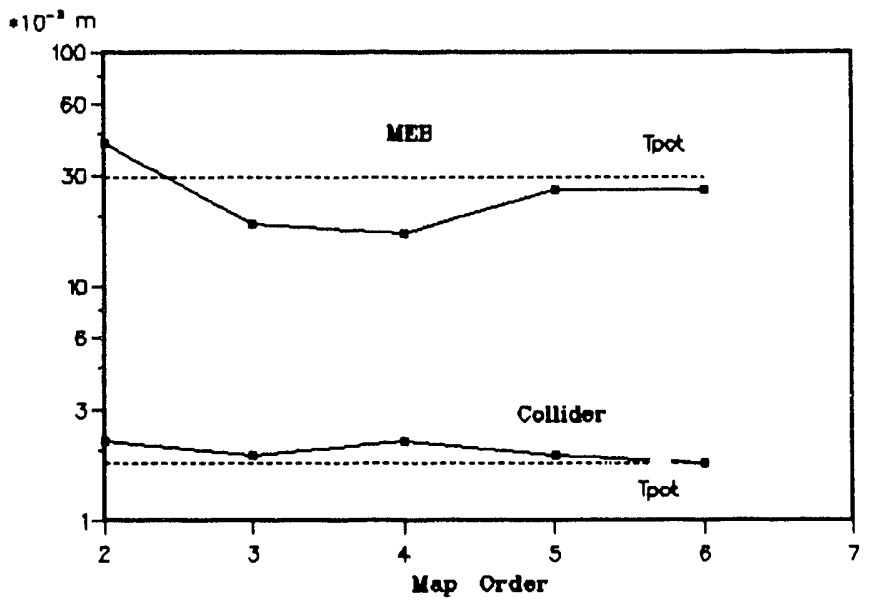

Figure 4. Dynamic apertures of the collider and MEB lattices calculated from map tracking.

defined differently in the two systems and thus were incompatible with one another for direct communication. We made some changes and created some subprograms that link both programs and could be read and executed by Teapot. We load Zlib in Teapot, so that Zlib's functions and subroutines could be directly called in Teapot. The following are some commands that we propose to be used by the Teapot user:

a) maptrk: a command similar to trackclo of Teapot. Its appearance switches the program to the "map mode" and it must be preceded by analysis command.

b) order $=$ : an integer number from 2 to 10 could be assigned to specify the map's order to be extracted or tracked.

c) mapname = : a string of characters of $\max$ size 16 could be assigned to specify the desired name for the map.

d) getzmap: a command for extracting the map of order in "order =" by using Zlib, and the absence of it means the map of the name in "mapname =" already exists so that the map extracting process could be skipped.

Here is an example of a section of Teapot input data: readfile

analysis, energy $=2.0 e 4, x t y p=1 . e-6, p x t y p=1 . e-06$, ytyp $=1 . e-06, p y t y p=1 . e-06, d p t y p=1 . e-6$, delta $=0.0000$

maptrk, energy $=2.0 e 4$, eigenamp, getzmap, order $=5$, mapname $=$ novImap5th

start, $x=1.4690 e-04, \quad p x=0.0, \quad y=1.4849 e-04, p y=0.0$, $d p=0.000$

start, $x=2.9380 e-04, p x=0.0, \quad y=2.8 ? 30 e-04, \quad p y=0.0$, $d p=0.000$

and so on; and the rest is the same as in the original Teapot. 


\section{CONCLUSION}

The imn' 'mentation of Zlib in Teapot gives the user more choices when tracking particles. Thin-element tracking gives more accurate results, and is convenient when dealing with most accelerator lattices for routine analysis. Fast map tracking with Zlib is now also available in an easy-to-use form, and our preliminary results indicate that for a well-corrected lattice, low order maps provide useful and effective perspectives about the accelerator lattices for efficient analysis of some of their properties.

\section{REFERENCES}

[1] Y. Yan, G. Bourianoff, and L. Schachinger, "A Typical 'Ztrack' Long-term Tracking Results for the SSC Aperture Study," Proc. of Workshop on Nonlinear Problems in Future Part. Accelerators, Capri, Italy, p. 297 (1990).
[2] B. Cole, G. Bourianoff, F. Pilat, "Particle Orbit Tracking on a Parallel Computer: Hypertrack," IEEE Particle Accelerator Conference, San Francisco, p. 204 (1991).

[3] Y. Yan, "Applications of Differential Algebra to Single Particle Dynamics in Storage Rings," SSCL-500 (1991).

[4] Y. Yan, T. Sen, A. Chao, G. Bourianoff, A. Dragt, and E. Forest," Comment on Round-off errors and on One-Turn Taylor Maps," SSCL-301 (1990).

[5] Y. Yan and C. Yan, "Zlib - A Numerical Library for Differential Algebra," SSCL-300 (1990).

[6] L. Schachinger and R. Talman, "Teapot: A Thin-Element Accelerator Program for Optics and Tracking," Particle Accel. 22, 35 (1987). 
$\checkmark$
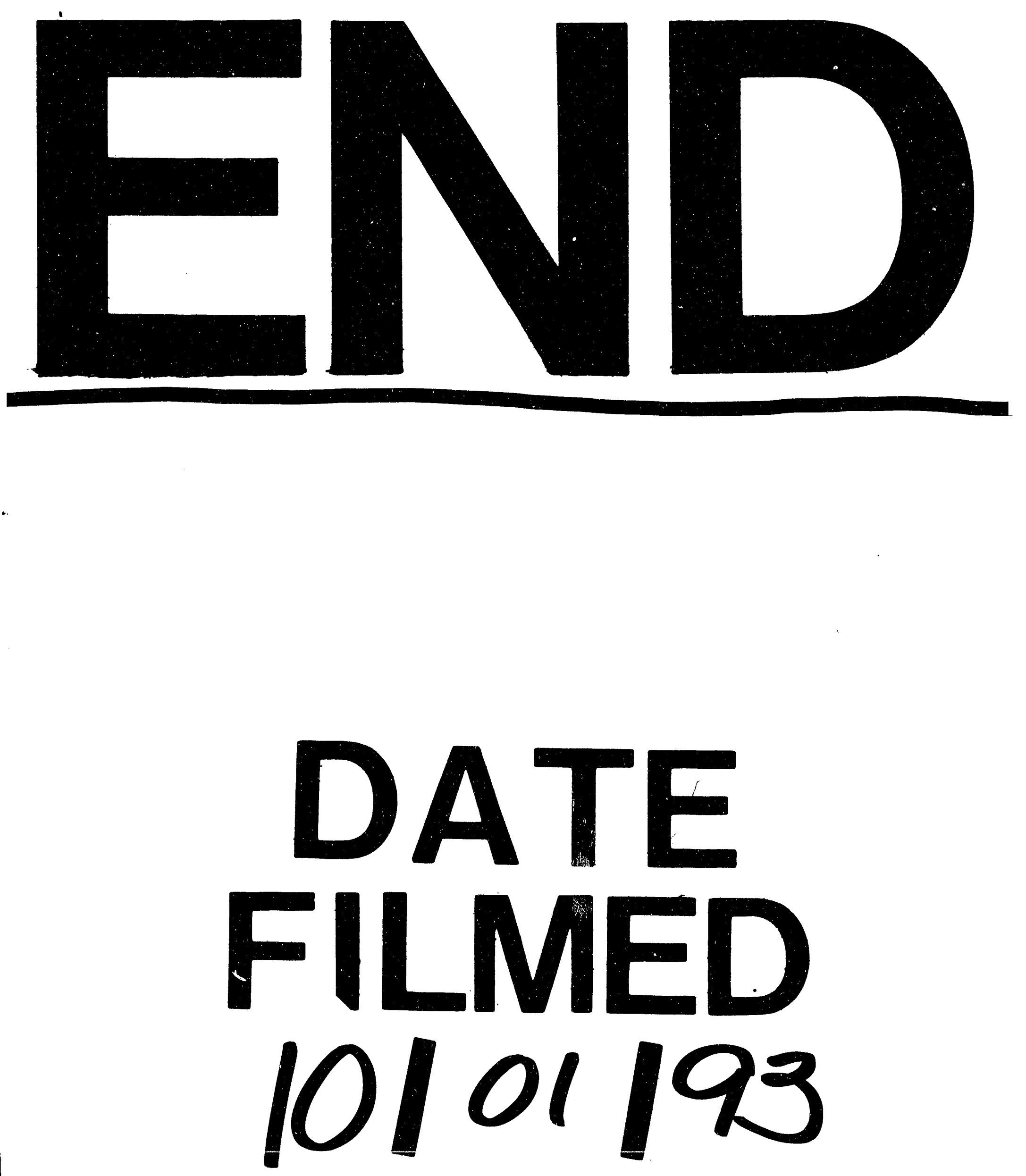

I 
